\title{
BASIC SETS FOR THE DOUBLE COVERING GROUPS OF THE SYMMETRIC AND ALTERNATING GROUPS IN ODD CHARACTERISTIC
}

\author{
OLIVIER BRUNAT AND JEAN-BAPTISTE GRAMAIN
}

\begin{abstract}
In this paper, following the methods of 9], we show that the double covering groups of the symmetric and alternating groups have $p$-basic sets for any odd prime $p$.
\end{abstract}

\section{INTRODUCTION}

Let $G$ be a finite group and $\mathcal{C}$ be a union of conjugacy classes of $G$. For any class function $\alpha$ on $G$, define $\operatorname{res}_{\mathcal{C}}(\alpha)=\widehat{\alpha}$ to be the class function on $G$ that takes the same values as $\alpha$ on $\mathcal{C}$ and 0 elsewhere. A $\mathcal{C}$-basic set of $G$ is a subset $b$ of the set $\operatorname{Irr}(G)$ of complex irreducible characters of $G$ such that $\widehat{b}=\{\widehat{\chi} \mid \chi \in b\}$ is a $\mathbb{Z}$-basis of the $\mathbb{Z}$-module generated by $\widehat{\operatorname{Irr}}(G)=\{\hat{\chi} \mid \chi \in \operatorname{Irr}(G)\}$.

In [7, §2.1], the authors develop a generalized modular theory of $G$ with respect to $\mathcal{C}$. In particular, there is a partition of $\operatorname{Irr}(G)$ into $\mathcal{C}$-blocks with respect to this $\mathcal{C}$-structure. We can then analogously define the notion of $\mathcal{C}$-basic set of any $\mathcal{C}$-block $B$. Note that, if $b$ is a $\mathcal{C}$-basic set of $G$ and $B$ is any $\mathcal{C}$-block of $G$, then $b \cap B$ is a $\mathcal{C}$-basic set of $B$. Conversely, given a $\mathcal{C}$-basic set for each $\mathcal{C}$-block of $G$, one recovers a $\mathcal{C}$-basic set of $G$.

Let $p$ be a prime. When $\mathcal{C}$ is the set of $p$-regular elements of $G$, that is, elements whose order is prime to $p$, the $\mathcal{C}$-modular theory of $G$ is the classical $p$-modular character theory, and the notion of $\mathcal{C}$-basic set coincides with that of $p$-basic set. One of the main challenges of Brauer's theory is the determination of the $p$-decomposition matrix of $G$, of which a first approximation can be obtained when one has a $p$-basic set of $G$.

While it is unclear how to exhibit a $p$-basic set of $G$ in general, Hiss conjectured that all finite groups do have $p$-basic sets. This conjecture is true in the following cases: for $p$-soluble groups [11, X.2.1], for certain finite reductive groups under various assumptions ([17], [12, [15, [13, [14], 22], [10, [6]), and for symmetric and alternating groups ([21, [9], [8], [2]).

The covering groups of the symmetric groups have a 2-basic set (see 4, Thm $5.2]$ ). For $p$ odd, attempts have been made in [3] and [1] to generalize the method of James and Kerber, but these methods fail for $p \geq 7$. In this paper, we prove the following.

Theorem 1.1. The covering groups of the symmetric and alternating groups have p-basic sets for any odd prime $p$.

2010 Mathematics Subject Classification. Primary 20C30, 20C15; Secondary 20C20.

The second author was supported by the EPSRC grant Combinatorial Representation Theory $\mathrm{EP} / \mathrm{M} 019292 / 1$. 
In [7] (see also [23]), the authors gave a notion of perfect isometries, which generalizes to $\mathcal{C}$ the perfect isometries introduced by Broué in [5]. Such a perfect isometry $I$ furthermore satisfies $I(\widehat{\chi})=\widehat{I(\chi)}$ for all $\chi \in B$, whence in particular sends a basic set to a basic set.

To prove Theorem 1.1, we follow the strategy of [9]. In Section 3, we present groups $\widetilde{G}$ and $\widetilde{H}$ and unions of conjugacy classes $\mathcal{C}$ of $\widetilde{G}$ and $\mathcal{D}$ of $\widetilde{H}$, and construct a $\mathcal{C}$-basic set of $\widetilde{G}$ (see Theorem 3.3 ) and a $\mathcal{D}$-basic set of $\widetilde{H}$ (see Theorem 3.4).

In Section 4, we adapt the perfect isometry constructed by Livesey in [24, where he proves Broué's Abelian Defect Perfect Isometry Conjecture, and we apply the methods and results of [7]. This produces, for any $p$-block $B$ of a double covering group of the symmetric or alternating groups, a perfect isometry between $B$ and a $\mathcal{C}$-block of $\widetilde{G}$ or a $\mathcal{D}$-block of $\widetilde{H}$, from which Theorem 1.1 is deduced. Finally, Section 5 is devoted to some further remarks. In particular, we recover some results of Olsson on the Brauer characters in $p$-blocks of covering groups ( $p$ odd), and their number.

\section{BACKGROUND}

Let $n$ be a positive integer. We write $\widetilde{\mathfrak{S}}_{n}$ for the double covering group of the symmetric group $\mathfrak{S}_{n}$ defined by

$\widetilde{\mathfrak{S}}_{n}=\left\langle z, t_{i}, 1 \leq i \leq n-1 \mid z^{2}=1, t_{i}^{2}=z,\left(t_{i} t_{i+1}\right)^{3}=z,\left(t_{i} t_{j}\right)^{2}=z(|i-j| \geq 2)\right\rangle$.

The group $\widetilde{\mathfrak{S}}_{n}$ and its representation theory were first studied by I. Schur in [31, and, unless otherwise specified, we always refer to 31 for details or proofs.

We recall that we have the following exact sequence

$$
1 \rightarrow\langle z\rangle \rightarrow \widetilde{\mathfrak{S}}_{n} \rightarrow \mathfrak{S}_{n} \rightarrow 1
$$

We denote by $\theta: \widetilde{\mathfrak{S}}_{n} \rightarrow \mathfrak{S}_{n}$ the natural projection. Note that for every $s \in \mathfrak{S}_{n}$, we have $\theta^{-1}(s)=\{\widetilde{s}, z \widetilde{s}\}$, where $\widetilde{s} \in \widetilde{\mathfrak{S}}_{n}$ is such that $\theta(\widetilde{s})=s$. Whenever $G$ is a subgroup of $\mathfrak{S}_{n}$, we set $\widetilde{G}=\theta^{-1}(G)$. Any irreducible (complex) character of $\widetilde{G}$ with $z$ in its kernel is simply lifted from an irreducible character of $G$. Any other irreducible character $\xi$ of $\widetilde{G}$ is called a spin character, and it satisfies $\xi(z)=-\xi(1)$. We denote by $\operatorname{SI}(\widetilde{G})$ the set of irreducible spin characters of $\widetilde{G}$. Define $\varepsilon=\operatorname{sgn} \circ \theta$, where sgn is the sign character of $\mathfrak{S}_{n}$. In particular, if $G$ is the alternating group $\mathcal{A}_{n}$, then $\widetilde{\mathcal{A}}_{n}=\operatorname{ker}(\varepsilon)$.

Note that the restriction of $\varepsilon$ to $\widetilde{G}$ (again denoted by $\varepsilon$ ) is a linear (irreducible) character of $\widetilde{G}$, and for any spin character $\xi$ of $\widetilde{G}, \varepsilon \otimes \xi$ is a spin character (because $\varepsilon \otimes \chi(z)=-\varepsilon \otimes \chi(1))$. A spin character $\xi$ is said to be self-associate if $\varepsilon \xi=\xi$. Otherwise, $\xi$ and $\varepsilon \xi$ are called associate characters.

If $H_{1}$ and $H_{2}$ are subgroups of $\mathfrak{S}_{n}$ acting non-trivially on disjoint subsets of $\{1, \ldots, n\}$ and $H=H_{1} \times H_{2} \leq \mathfrak{S}_{n}$, then Schur defined in [31] (see also [18]) the twisted central product denoted by $\hat{\times}$ and such that $\widetilde{H}=\widetilde{H}_{1} \hat{\times} \widetilde{H}_{2} \leq \widetilde{\mathfrak{S}}_{n}$. Schur proved that there is a surjective map $\hat{\otimes}: \operatorname{SI}\left(\widetilde{H}_{1}\right) \times \operatorname{SI}\left(\widetilde{H}_{2}\right) \rightarrow \operatorname{SI}(\widetilde{H})$. Schur explicitly described when two pairs of spin characters have the same image $\chi \hat{\otimes} \psi$ under this map, and how to detect whether $\chi \hat{\otimes} \psi$ is self-associate or not. Furthermore, we derive from case 1 of $\S 2$ and the proof of Theorem 2.4 of [18] that 
Proposition 2.1. Let $\widetilde{H}_{1}$ and $\widetilde{H}_{2}$ be as above and not contained in the kernel of $\varepsilon$. Let $\chi_{1}$ and $\chi_{2}$ be irreducible spin characters of $\widetilde{H}_{1}$ and $\widetilde{H}_{2}$. Let $\tau_{1} \in \widetilde{H}_{1}$ and $\tau_{2} \in \widetilde{H}_{2}$ be such that $\varepsilon\left(\tau_{1}\right)=\varepsilon\left(\tau_{2}\right)=1$. Then

(1) If $\chi_{1}$ (or $\chi_{2}$ ) is self-associate, then

$$
\left(\chi_{1} \hat{\otimes} \chi_{2}\right)\left(\tau_{1}, \tau_{2}\right)=\chi_{1}\left(\tau_{1}\right) \chi_{2}\left(\tau_{2}\right) .
$$

(2) If $\chi_{1}$ and $\chi_{2}$ are non-self-associate, then

$$
\left(\chi_{1} \hat{\otimes} \chi_{2}\right)\left(\tau_{1}, \tau_{2}\right)=2 \chi_{1}\left(\tau_{1}\right) \chi_{2}\left(\tau_{2}\right) .
$$

Let $\mathcal{D}_{n}$ be the set of bar partitions of $n$, i.e. the partitions of $n$ with distinct parts. Denote by $\mathcal{D}_{n}^{+}$(respectively $\mathcal{D}_{n}^{-}$) the subset of $\mathcal{D}_{n}$ consisting of all partitions $\pi \in \mathcal{D}_{n}$ such that the number of even parts of $\pi$ is even (respectively odd). In 31, Schur proved that the spin characters of $\widetilde{\mathfrak{S}}_{n}$ are, up to association, labeled by $\mathcal{D}_{n}$. More precisely, he showed that every $\lambda \in \mathcal{D}_{n}^{+}$indexes a self-associate spin character $\xi_{\lambda}$, and every $\lambda \in \mathcal{D}_{n}^{-}$a pair $\left(\xi_{\lambda}^{+}, \xi_{\lambda}^{-}\right)$of associate spin characters.

The irreducible spin characters of $\widetilde{\mathcal{A}}_{n}$ are also labeled by the bar partitions of $n$. If $\lambda \in \mathcal{D}_{n}^{+}$, then $\operatorname{Res}_{\widetilde{\mathcal{A}}_{n}}^{\widetilde{\widetilde{S}}_{n}}\left(\xi_{\lambda}\right)=\zeta_{\lambda}^{+}+\zeta_{\lambda}^{-}$with $\zeta_{\lambda}^{+}, \zeta_{\lambda}^{-} \in \operatorname{SI}\left(\widetilde{\mathcal{A}}_{n}\right)$. If $\lambda \in \mathcal{D}_{n}^{-}$, then $\operatorname{Res} \widetilde{\mathcal{A}}_{n}^{\widetilde{\mathcal{S}}_{n}}\left(\xi_{\lambda}^{+}\right)=\operatorname{Res} \widetilde{\mathcal{A}}_{n}^{\widetilde{\mathcal{S}}_{n}}\left(\xi_{\lambda}^{-}\right)=\zeta_{\lambda} \in \operatorname{SI}\left(\widetilde{\mathcal{A}}_{n}\right)$.

For any bar partition $\lambda=\left(\lambda_{1}>\cdots>\lambda_{k}>0\right)$ of $n$, we set, as in the case of partitions, $|\lambda|=\sum \lambda_{i}$ and we define the length $\ell(\lambda)$ of $\lambda$ by $\ell(\lambda)=k$. Furthermore, we set $\sigma(\lambda)=(-1)^{|\lambda|-\ell(\lambda)}$. With this notation, we then have $\lambda \in \mathcal{D}_{n}^{\sigma(\lambda)}$ (see e.g. [30, p. 45]).

Let $p$ be an odd integer. To any $\lambda \in \mathcal{D}_{n}$, one can associate in a canonical way its $\bar{p}$-core $\lambda_{(\bar{p})}$ and its $\bar{p}$-quotient $\lambda^{(\bar{p})}$; see [30, p. 28]. The $\bar{p}$-core $\lambda_{(\bar{p})}$ is a bar partition of $n-w p$, where $w$ is a non-negative integer called the $\bar{p}$-weight of $\lambda$. The $\bar{p}$-quotient has the form $\lambda^{(\bar{p})}=\left(\lambda^{0}, \lambda^{1}, \ldots, \lambda^{(p-1) / 2}\right)$, where $\lambda^{0}$ is a bar partition, the $\lambda^{i}$ 's are partitions for $1 \leq i \leq(p-1) / 2$, and the sizes of the $\lambda_{i}$ 's $(0 \leq i \leq(p-1) / 2)$ add up to $w$. If we write, in analogy with bar partitions, $\sigma\left(\lambda^{(\bar{p})}\right)=(-1)^{w-\ell\left(\lambda^{0}\right)}$, then we obtain that

$$
\sigma(\lambda)=\sigma\left(\lambda_{(\bar{p})}\right) \sigma\left(\lambda^{(\bar{p})}\right)
$$

\section{SOME BASIC SETS}

Let $w$ be a positive integer and $p$ be an odd prime. In this section, we will focus on a group $\widetilde{G}$ which, when $w<p$, is isomorphic to the normalizer of a Sylow $p$-subgroup of $\widetilde{\mathfrak{S}}_{p w}$. We will recall the description of its irreducible characters, and describe a union $\mathcal{C}$ of conjugacy classes and a $\mathcal{C}$-basic set for $\widetilde{G}$.

Set $K=\mathbb{Z} / p \mathbb{Z} \rtimes \mathbb{Z} /(p-1) \mathbb{Z}$ and $G=K \imath \mathfrak{S}_{w}$. Recall that $G$ is a natural subgroup of $\mathfrak{S}_{p w}$ and that $G=N \rtimes \mathfrak{S}_{w}$, where $N=K^{w}$. Now, let $Q=(\mathbb{Z} / p \mathbb{Z})^{w} \triangleleft N$ and $L=\mathbb{Z} /(p-1) \mathbb{Z} \imath \mathfrak{S}_{w}$. Then $G=Q \rtimes L$. Note that $|\widetilde{Q}|=2 p^{w}$. Thus, by Sylow's theorems, $\widetilde{Q}$ has a Sylow $p$-subgroup $P$ of index 2 , whence normal and unique in $\widetilde{Q}$. Since $\langle z\rangle$ is normal in $\widetilde{Q}$ and $\langle z\rangle \cap P$ is trivial (as $p$ is odd), we see that $\widetilde{Q}=\langle z\rangle \times P$ and that $P \simeq Q$. Now take any $g \in \widetilde{G}$. Then, since $\widetilde{Q} \triangleleft \widetilde{G}$, the group $g P g^{-1}$ is a 
Sylow $p$-subgroup of $\widetilde{Q}$. Hence, $g P g^{-1}=P$ and $P$ is normal in $\widetilde{G}$. Furthermore, as $Q \cap L=1$, we have $P \cap \widetilde{L}=1$ (because $P \cap \widetilde{L}=P \cap(\widetilde{Q} \cap \widetilde{L})=P \cap\langle z\rangle=1$ ). Hence

$$
\widetilde{G}=\widetilde{N} \widetilde{\mathfrak{S}}_{w}=P \rtimes \widetilde{L} .
$$

The irreducible spin characters of $\widetilde{G}$ were constructed by Michler and Olsson in 25. using the Clifford's theory (see for example [20, Chap 6]) of $\widetilde{G}$ with respect to the normal subgroup $\widetilde{N}$. The parametrization they obtain is as follows.

Proposition 3.1. The irreducible spin characters of $\widetilde{G}$ can be labeled explicitly by the $\bar{p}$-quotients of $w$. If $q=\left(q^{0}, q^{1}, \ldots, q^{(p-1) / 2}\right)$ is a $\bar{p}$-quotient of $w$, then $q$ labels two associate characters $\psi_{q}^{+}$and $\psi_{q}^{-}$if and only if $\sigma(q)=-1$; otherwise, $q$ labels a unique self-associate character $\psi_{q}$.

For the second statement, an explanation can be found for example in [16, p. 422].

Proposition 3.2. The spin characters of $\widetilde{G}$ which have $P$ in their kernel are exactly those labeled by $\bar{p}$-quotients of the form $q=\left(\emptyset, q^{1}, \ldots, q^{(p-1) / 2}\right)$.

Proof. Take any $\psi \in \operatorname{Irr}(\widetilde{G})$. Since $P \leq \tilde{N}$, to study the restriction of $\psi$ to $P$, we will look at the restriction of $\psi$ to $\widetilde{N}$. On the other hand, the restriction to $\widetilde{N}$ is given by Clifford's theorem [20, Thm 6.2] which goes as follows. There exist a unique $\widetilde{G}$-orbit $\Omega_{\psi}$ of irreducible characters of $\widetilde{N}$ and a unique positive integer $e_{\psi}$ such that

$$
\operatorname{Res}_{\widetilde{N}}^{\widetilde{G}}(\psi)=e_{\psi} \sum_{\chi \in \Omega_{\psi}} \chi
$$

It follows from (3), the facts that $\psi$ is a spin character and that all $\chi \in \Omega_{\psi}$ take the same value on $z$ that $\Omega_{\psi}$ consists only of spin characters. We will thus now recall the description given in [25] of the $\widetilde{G}$-orbits of spin characters of $\widetilde{N}$.

The irreducible spin characters of $\widetilde{N}$ are of the form $\chi_{1} \hat{\otimes} \cdots \hat{\otimes} \chi_{w}$, where $\chi_{i} \in$ $\operatorname{SI}(\widetilde{K})$ for $1 \leq i \leq w$. As above, we have $\widetilde{K}=\langle\tau\rangle \rtimes \theta^{-1}(\mathbb{Z} /(p-1) \mathbb{Z})$, where $\tau$ is an element or order $p$. In [25], Michler and Olsson show that $\widetilde{K}$ has exactly $p$ spin characters: a unique self-associate $\eta_{0}$, of degree $p-1$ and with $\eta_{0}(\tau)=-1$, and $(p-1) / 2$ pairs of associate linear characters $\eta_{1}^{+}, \eta_{1}^{-}, \ldots, \eta_{(p-1) / 2}^{+}, \eta_{(p-1) / 2}^{-}$, which are exactly those having $\langle\tau\rangle$ in their kernel.

For any decomposition $t=\left(t_{0}, t_{1}, \ldots, t_{(p-1) / 2}\right)$ of $w$, we set

$$
\theta_{t}=\underbrace{\eta_{0} \hat{\otimes} \cdots \hat{\otimes} \eta_{0}}_{t_{0} \text { factors }} \hat{\otimes} \underbrace{\eta_{1}^{+} \hat{\otimes} \cdots \hat{\otimes} \eta_{1}^{+}}_{t_{1} \text { factors }} \hat{\otimes} \cdots \hat{\otimes} \underbrace{\eta_{(p-1) / 2}^{+} \hat{\otimes} \cdots \hat{\otimes} \eta_{(p-1) / 2}^{+}}_{t_{(p-1) / 2} \text { factors }} \in \operatorname{SI}(\tilde{N}) .
$$

Now suppose $\psi$ is labeled by the $\bar{p}$-quotient $q=\left(q^{0}, \ldots, q^{(p-1) / 2}\right)$ of $w$. From 25 , Prop 3.12] and the construction of $\psi$, we get that, if $\left|q^{0}\right|>1$, then $\theta_{\left(\left|q^{0}\right|, \ldots,\left|q^{(p-1) / 2}\right|\right)}$ is a representative for $\Omega_{\psi}=\Omega_{\varepsilon \psi}$ (even if $\psi \neq \varepsilon \psi$ ). If $\left|q^{0}\right| \leq 1$ and $w-\left|q^{0}\right|$ is odd, then $\psi \neq \varepsilon \psi$ and representatives for $\Omega_{\psi}$ and $\Omega_{\varepsilon \psi}$ are given by $\theta_{\left(\left|q^{0}\right|, \ldots,\left|q^{(p-1) / 2}\right|\right)}$ and $\varepsilon \theta_{\left(\left|q^{0}\right|, \ldots,\left|q^{(p-1) / 2}\right|\right)}$ (not necessarily in this order). In all cases, we write $\theta_{\psi}$ for the representative of $\Omega_{\psi}$.

Note that $Q=\left\langle u_{1}\right\rangle \times \cdots \times\left\langle u_{w}\right\rangle$, where $u_{i}$ has order $p$ for $1 \leq i \leq w$. For $1 \leq i \leq w$, let $\tau_{i}$ be the unique element of order $p$ in $\theta^{-1}\left(\left\{u_{i}\right\}\right)$. Then

$$
P=\left\langle\tau_{1}\right\rangle \times \cdots \times\left\langle\tau_{w}\right\rangle .
$$


In particular, $P \leq \widetilde{\mathcal{A}}_{p w}$.

We now have $\psi(1)=e_{\psi} \sum_{\chi \in \Omega_{\psi}} \chi(1)=e_{\psi} \theta_{\psi}(1)\left|\Omega_{\psi}\right|$. Furthermore, iterating Proposition 2.1 shows that, for any $\chi \in \Omega_{\psi}$, there is a non-negative integer $\alpha_{\chi}$ such that $\chi(1)=2^{\alpha_{\chi}}(p-1)^{\left|q^{0}\right|}$ and $\chi\left(\tau_{1}, \ldots, \tau_{w}\right)=2^{\alpha_{\chi}}(-1)^{\left|q^{0}\right|}$. Since $\chi(1)$ does not depend on the choice of $\chi$ in $\Omega_{\psi}$, neither does $\alpha_{\chi}$ which we thus denote by $\alpha$. In particular, we have

$$
\psi(1)=e_{\psi} 2^{\alpha}(p-1)^{\left|q^{0}\right|}\left|\Omega_{\psi}\right| \quad \text { and } \quad \psi\left(\tau_{1} \cdots \tau_{w}\right)=e_{\psi} 2^{\alpha}(-1)^{\left|q^{0}\right|}\left|\Omega_{\psi}\right| .
$$

It follows that, if $q^{0} \neq \emptyset$, then $\psi\left(\tau_{1} \cdots \tau_{w}\right) \neq \psi(1)$, whence $P$ is not contained in $\operatorname{ker}(\psi)$. If $q^{0}=\emptyset$, then a similar computation shows that, for any $\left(x_{1}, \ldots, x_{w}\right) \in P$, we have $\psi\left(x_{1} \cdots x_{w}\right)=e_{\psi} 2^{\alpha}\left|\Omega_{\psi}\right|=\psi(1)$, as required.

We define $\mathcal{C}$ to be the union of conjugacy classes of $\widetilde{G}$ which have a representative in $\widetilde{L}$.

Theorem 3.3. The set of irreducible spin characters of $\widetilde{G}$ is a union of $\mathcal{C}$-blocks, with $\mathcal{C}$-basic set the set $B$ of spin characters labeled by $\bar{p}$-quotients of the form $q=\left(\emptyset, q^{1}, \ldots, q^{(p-1) / 2}\right)$.

Proof. Let $\chi$ be a non-spin character of $\widetilde{G}$ and $\psi$ be a spin character of $\widetilde{G}$. For any $g \in \widetilde{G}$, one has $\chi(g)=\chi(z g)$ and $\psi(g)=-\psi(z g)$. Also, since $z \in \widetilde{L}$, we have that $\mathcal{C}=z \mathcal{C}$. Thus, writing, for any $A \subseteq \widetilde{G},\langle\psi, \chi\rangle_{A}=\frac{1}{|\widetilde{G}|} \sum_{x \in A} \psi(x) \overline{\chi(x)}$, we have

$$
2|\widetilde{G}|\langle\psi, \chi\rangle_{\mathcal{C}}=|\widetilde{G}|\left(\langle\psi, \chi\rangle_{\mathcal{C}}+\langle\psi, \chi\rangle_{z \mathcal{C}}\right)=\sum_{g \in \mathcal{C}}(\psi(g)+\psi(z g) \overline{\chi(g)}=0 .
$$

This proves that the set of spin characters of $\widetilde{G}$ is a union of $\mathcal{C}$-blocks (see [7, Prop 2.14 ] and [23, §1]). By Lemma 4.1 and Proposition 4.2 of [9], the set of irreducible characters of $\widetilde{G}$ with $P$ in their kernel is a $\mathcal{C}$-basic set of $\widetilde{G}$. The intersection of this basic set with the set of spin characters of $\widetilde{G}$, which is given by Proposition 3.2, is thus a $\mathcal{C}$-basic set for the set of spin characters of $\widetilde{G}$.

Let $H=G \cap \mathcal{A}_{p w}$. By Clifford's theory between $\widetilde{G}$ and $\widetilde{H}$, the spin irreducible characters of $\widetilde{H}$ are again labeled by the $\bar{p}$-quotients of $w$. Any $\bar{p}$-quotient $q$ of $w$ labels two characters $\varphi_{q}^{+}$and $\varphi_{q}^{-}$of $\widetilde{H}$ when $\sigma(q)=1$, and one character $\varphi_{q}$ otherwise. Set $\mathcal{D}=\mathcal{C} \cap \widetilde{H}$.

Theorem 3.4. The set of irreducible spin characters of $\widetilde{H}$ is a union of $\mathcal{D}$-blocks, with $\mathcal{D}$-basic set the set $B^{\prime}$ of spin characters labeled by $\bar{p}$-quotients of the form $q=\left(\emptyset, q^{1}, \ldots, q^{(p-1) / 2}\right)$.

Proof. Since $z$ lies in $\mathcal{D}$, we conclude as in Theorem 3.3 that the set of irreducible spin characters of $\widetilde{H}$ is a union of $\mathcal{D}$-blocks. Furthermore, a simple counting argument shows that

$$
\widetilde{H}=P \rtimes\left(\widetilde{L} \cap \widetilde{\mathcal{A}}_{p w}\right) .
$$

Let $q=\left(q^{0}, q^{1}, \ldots, q^{(p-1) / 2}\right)$ be a $\bar{p}$-quotient of $w$. If $\sigma(q)=-1$, then $\varphi_{q}=$ $\operatorname{Res}_{\widetilde{H}}^{\widetilde{G}}\left(\psi_{q}^{+}\right)$has $P$ in its kernel if and only if $\psi_{q}^{+}$does. If $\sigma(q)=1$, then $\operatorname{Res}_{\widetilde{H}}^{\widetilde{G}}\left(\psi_{q}\right)=$ $\varphi_{q}^{+}+\varphi_{q}^{-}$, with $\varphi_{q}^{+}(1)=\varphi_{q}^{-}(1)=\psi_{q}(1) / 2$. Let $x \in P$. We have $\psi_{q}(x)=\psi_{q}(1)$ if and only if $\varphi_{q}^{+}(x)+\varphi_{q}^{-}(x)=\psi_{q}(1)$; since, by [20, Lemma 2.15], $\left|\varphi_{q}^{+}(x)\right| \leq \psi_{q}(1) / 2$ and $\left|\varphi_{q}^{-}(x)\right| \leq \psi_{q}(1) / 2$, this holds if and only if $\varphi_{q}^{+}(x)=\varphi_{q}^{-}(x)=\psi_{q}(1) / 2$. 
This proves that any spin character of $\widetilde{H}$ labeled by $q$ has $P$ in its kernel if and only if $q^{0}=\emptyset$, and we can conclude as in the proof of Theorem 3.3

Remark 3.5. The same arguments as in the proofs of Theorems 3.3 and 3.4 show that the set of characters of $G$ with $Q$ in their kernel is a $\theta(\mathcal{C})$-basic set of $G$ (which is the same as that found in [9]), and that the set of characters of $H$ with $Q$ in their kernel is a $\theta(\mathcal{D})$-basic set of $H$.

\section{PERfECT ISOMETRIES AND BASIC SETS FOR THE COVERING GROUPS}

We start by recalling the definitions of perfect isometry and of Broué isometry, and we prove some of their properties which will be useful later.

Let $G$ and $G^{\prime}$ be two finite groups, and $\mathcal{C}$ and $\mathcal{C}^{\prime}$ unions of conjugacy classes of $G$ and $G^{\prime}$ respectively. Let $B$ (resp. $B^{\prime}$ ) be a union of $\mathcal{C}$-blocks of $G$ (resp. of $\mathcal{C}^{\prime}$-blocks of $\left.G^{\prime}\right)$; see [7, p.4]. An isometry $I: \mathbb{C} B \rightarrow \mathbb{C} B^{\prime}$ such that $I(\mathbb{Z} B)=\mathbb{Z} B^{\prime}$ is a perfect isometry if

$$
I \circ \operatorname{res}_{\mathcal{C}}=\operatorname{res}_{\mathcal{C}^{\prime}} \circ I
$$

Remark 4.1. If $I: \mathbb{C} B \rightarrow \mathbb{C} B^{\prime}$ and $J: \mathbb{C} B^{\prime} \rightarrow \mathbb{C} B^{\prime \prime}$ are perfect isometries, then it follows easily from (5) that $J \circ I: \mathbb{C} B \rightarrow \mathbb{C} B^{\prime \prime}$ is a perfect isometry.

To $I$, we associate $\widehat{I} \in \mathbb{C}\left(B \times B^{\prime}\right)$ as in [7, $\left.\S 2.3\right]$ which satisfies

$$
\widehat{I}=\sum_{\chi \in B} \bar{\chi} \otimes I(\chi) \text {. }
$$

Recall that (see [7, $\operatorname{Eq}(16)]$ ), for any $\psi \in \mathbb{C} \operatorname{Irr}(B)$ and $y \in G^{\prime}$, we have

$$
I(\psi)(y)=\frac{1}{|G|} \sum_{x \in G} \widehat{I}(x, y) \psi(x) .
$$

Using (6) and (7), an easy computation shows that, if $B^{\prime \prime}$ is a union of $\mathcal{C}^{\prime \prime}$-blocks of $G^{\prime \prime}$ and $J: \mathbb{C} B^{\prime} \rightarrow \mathbb{C} B^{\prime \prime}$ is a perfect isometry, then for any $(x, z) \in G \times G^{\prime \prime}$

$$
\widehat{J \circ I}(x, z)=\frac{1}{\left|G^{\prime}\right|} \sum_{y \in G^{\prime}} \widehat{I}(x, y) \widehat{J}(y, z) .
$$

If $p$ is a prime and $(K, \mathcal{R}, k)$ is a splitting $p$-modular system for $G$ and $G^{\prime}$, and if $\mathcal{C}$ and $\mathcal{C}^{\prime}$ are the sets of $p$-regular elements of $G$ and $G^{\prime}$, then $I$ is a Broue isometry if the following two properties are satisfied.

(i) For every $\left(x, x^{\prime}\right) \in G \times G^{\prime}, \widehat{I}\left(x, x^{\prime}\right)$ lies in $\left|\mathrm{C}_{G}(x)\right| \mathcal{R} \cap\left|\mathrm{C}_{G^{\prime}}\left(x^{\prime}\right)\right| \mathcal{R}$.

(ii) If $\widehat{I}\left(x, x^{\prime}\right) \neq 0$, then $x$ and $x^{\prime}$ are either both $p$-regular or both $p$-singular.

Note that, in this case, $I$ is also a perfect isometry in the above sense since (ii) implies (5).

Lemma 4.2. If $I: \mathbb{C} B \rightarrow \mathbb{C} B^{\prime}$ and $J: \mathbb{C} B^{\prime} \rightarrow \mathbb{C} B^{\prime \prime}$ are Broué isometries, then $J \circ I: \mathbb{C} B \rightarrow \mathbb{C} B^{\prime \prime}$ is a Broué isometry.

Proof. Take any $x \in G$ and $z \in G^{\prime \prime}$. First note that $y \mapsto \widehat{I}(x, y) \widehat{J}(y, z)$ is a class function of $G^{\prime}$. Thus, by (8),

$$
\frac{\widehat{J \circ I}(x, z)}{\left|\mathrm{C}_{G}(x)\right|}=\sum_{y \in G^{\prime} / \sim} \frac{\widehat{I}(x, y)}{\left|\mathrm{C}_{G}(x)\right|} \frac{\widehat{J}(y, z)}{\left|\mathrm{C}_{G^{\prime}}(y)\right|} \in \mathcal{R} .
$$


A similar argument shows that $\widehat{J \circ I}(x, z) \in\left|\mathrm{C}_{G}(z)\right| \mathcal{R}$, whence $\widehat{J \circ I}$ satisfies (i). Now suppose $\widehat{J \circ I}(x, z) \neq 0$. Then, by (주), there exists $y \in G^{\prime}$ such that $\widehat{I}(x, y) \neq 0$ and $\widehat{J}(y, z) \neq 0$; (ii) follows.

Remark 4.3. Given a Broué isometry $I$, a formula for $\widehat{I^{-1}}$ can be found in [7, Remark 2.12]. It easily implies that $I^{-1}$ is a Broué isometry.

Theorem 4.4. Let $p$ be an odd prime and $B$ be a p-block of $\widetilde{\mathfrak{S}}_{n}$ such that $\left\{\xi_{\lambda}^{+}, \xi_{\lambda}^{-}\right\} \subseteq$ $B$ for some $\lambda \in \mathcal{D}_{n}^{-}$. Let $J: \mathbb{C} B \rightarrow \mathbb{C} B$ be the isometry given by $J\left(\xi_{\lambda}^{+}\right)=\xi_{\lambda}^{-}$, $J\left(\xi_{\lambda}^{-}\right)=\xi_{\lambda}^{+}$, and $J(\xi)=\xi$ if $\xi \notin\left\{\xi_{\lambda}^{+}, \xi_{\lambda}^{-}\right\}$. Then $J$ is a Broué isometry.

Proof. Recall that a set of conjugacy classes of $\widetilde{\mathfrak{S}}_{n}$ (the split classes) outside which all spin characters vanish can be labeled by the elements of $\mathcal{O}_{n} \cup \mathcal{D}_{n}^{-}$, where $\mathcal{O}_{n}$ is the set of partitions of $n$ in odd parts. Each $\pi \in \mathcal{O}_{n} \cup \mathcal{D}_{n}^{-}$labels two conjugacy classes with representatives $t_{\pi}$ and $z t_{\pi}$ such that the following hold. For all $\pi \in \mathcal{O}_{n}$, $\xi_{\lambda}^{+}\left(t_{\pi}\right)=\xi_{\lambda}^{-}\left(t_{\pi}\right)$ and $\xi_{\lambda}^{+}\left(z t_{\pi}\right)=\xi_{\lambda}^{-}\left(z t_{\pi}\right)$. For all $\pi \in \mathcal{D}_{n}^{-}$,

$$
\xi_{\lambda}^{+}\left(t_{\pi}\right)=\xi_{\lambda}^{-}\left(z t_{\pi}\right)=-\xi_{\lambda}^{+}\left(z t_{\pi}\right)=-\xi_{\lambda}^{-}\left(t_{\pi}\right)=\delta_{\pi \lambda} i^{(n-k+1) / 2} \sqrt{z_{\lambda} / 2},
$$

where $\lambda=\left(\lambda_{1}>\cdots>\lambda_{k}>0\right)$ and $z_{\lambda}=\lambda_{1} \cdots \lambda_{k}$.

Denote by $I$ the identity on $\mathbb{C} B$. By [7, Theorem 4.21], $I$ is a Broué isometry. First suppose $\pi=\pi^{\prime}=\lambda$ and take $\nu \in\{0,1\}$. Then, by ([6),

$$
\begin{aligned}
\widehat{J}\left(t_{\lambda}, z^{\nu} t_{\lambda}\right)-\widehat{I}\left(t_{\lambda}, z^{\nu} t_{\lambda}\right)= & \left(\overline{\xi_{\lambda}^{+}\left(t_{\lambda}\right)} \xi_{\lambda}^{-}\left(z^{\nu} t_{\lambda}\right)+\overline{\xi_{\lambda}^{-}\left(t_{\lambda}\right)} \xi_{\lambda}^{+}\left(z^{\nu} t_{\lambda}\right)\right) \\
& -\left(\xi_{\lambda}^{+}\left(t_{\lambda}\right) \xi_{\lambda}^{+}\left(z^{\nu} t_{\lambda}\right)+\xi_{\lambda}^{-}\left(t_{\lambda}\right) \xi_{\lambda}^{-}\left(z^{\nu} t_{\lambda}\right)\right) .
\end{aligned}
$$

If $(n-k+1) / 2$ is odd, then $\overline{\xi_{\lambda}^{+}\left(t_{\lambda}\right)}=\xi_{\lambda}^{-}\left(t_{\lambda}\right)$ and $\overline{\xi_{\lambda}^{-}\left(t_{\lambda}\right)}=\xi_{\lambda}^{+}\left(t_{\lambda}\right)$, so that $\widehat{J}\left(t_{\lambda}, z^{\nu} t_{\lambda}\right)-\widehat{I}\left(t_{\lambda}, z^{\nu} t_{\lambda}\right)=0$. Thus (i) and (ii) hold for $\widehat{J}$ (and $x=t_{\lambda}, x^{\prime}=z^{\nu} t_{\lambda}$ ).

$$
\begin{aligned}
& \text { If }(n-k+1) / 2 \text { is even, then } \overline{\xi_{\lambda}^{+}\left(t_{\lambda}\right)}=\xi_{\lambda}^{+}\left(t_{\lambda}\right) \text { and } \overline{\xi_{\lambda}^{-}\left(t_{\lambda}\right)}=\xi_{\lambda}^{-}\left(t_{\lambda}\right) \text {, so that } \\
& \widehat{J}\left(t_{\lambda}, z^{\nu} t_{\lambda}\right)-\widehat{I}\left(t_{\lambda}, z^{\nu} t_{\lambda}\right)=\left(\xi_{\lambda}^{+}\left(t_{\lambda}\right)-\xi_{\lambda}^{-}\left(t_{\lambda}\right)\right)\left(\xi_{\lambda}^{-}\left(z^{\nu} t_{\lambda}\right)-\xi_{\lambda}^{+}\left(z^{\nu} t_{\lambda}\right)\right) \\
& =2 \xi_{\lambda}^{+}\left(t_{\lambda}\right) 2 \xi_{\lambda}^{-}\left(z^{\nu} t_{\lambda}\right) \\
& =(-1)^{\nu+1} 4 \xi_{\lambda}^{+}\left(t_{\lambda}\right)^{2} \\
& = \pm 2 z_{\lambda} \text {. }
\end{aligned}
$$

Since $2 z_{\lambda}=\left|\mathrm{C}_{\widetilde{\mathfrak{S}}_{n}}\left(t_{\lambda}\right)\right|$, this shows that (i) holds for $\widehat{J}$ (and $x=t_{\lambda}, x^{\prime}=z^{\nu} t_{\lambda}$ ). Note that (ii) is automatic in this case since $t_{\lambda}$ and $z^{\nu} t_{\lambda}$ are both $p$-regular or both $p$-singular.

Suppose now that $\pi, \pi^{\prime} \in \mathcal{O}_{n} \cup \mathcal{D}_{n}^{-} \backslash\{\lambda\}$ and $\nu, \nu^{\prime} \in\{0,1\}$. Then

$$
\widehat{J}\left(z^{\nu} t_{\pi}, z^{\nu^{\prime}} t_{\pi^{\prime}}\right)=\widehat{I}\left(z^{\nu} t_{\pi}, z^{\nu^{\prime}} t_{\pi^{\prime}}\right) .
$$

In particular, (i) and (ii) are true for $\widehat{J}$ in this case.

Finally, take $x \in \widetilde{\mathfrak{S}}_{n}$ such that exactly one of $x$ and $t_{\lambda}$ is $p$-regular. Then $\widehat{I}\left(x, t_{\lambda}\right)=0$. On the other hand,

$$
\begin{aligned}
\widehat{J}\left(x, t_{\lambda}\right)-\widehat{I}\left(x, t_{\lambda}\right) & =\left(\overline{\xi_{\lambda}^{+}(x)} \xi_{\lambda}^{-}\left(t_{\lambda}\right)+\overline{\xi_{\lambda}^{-}(x)} \xi_{\lambda}^{+}\left(t_{\lambda}\right)\right)-\left(\overline{\xi_{\lambda}^{+}(x)} \xi_{\lambda}^{+}\left(t_{\lambda}\right)+\overline{\xi_{\lambda}^{-}(x)} \xi_{\lambda}^{-}\left(t_{\lambda}\right)\right) \\
& =\left(\overline{\xi_{\lambda}^{+}(x)}-\overline{\xi_{\lambda}^{-}(x)}\right)\left(\xi_{\lambda}^{-}\left(t_{\lambda}\right)-\xi_{\lambda}^{+}\left(t_{\lambda}\right)\right),
\end{aligned}
$$


and $\overline{\xi_{\lambda}^{+}(x)}-\overline{\xi_{\lambda}^{-}(x)}=0$ since $x$ is conjugate neither to $t_{\lambda}$ nor to $z t_{\lambda}$. Hence $\widehat{J}\left(x, t_{\lambda}\right)=$ 0 , and (i) and (ii) hold in this case. More generally, whenever $x \notin\left\{t_{\lambda}, z t_{\lambda}\right\}$ and $\nu \in\{0,1\}$,

$$
\widehat{J}\left(x, z^{\nu} t_{\lambda}\right)=\widehat{I}\left(x, z^{\nu} t_{\lambda}\right),
$$

whence (i) and (ii) hold. The result follows.

From now on, we fix an integer $n \geq 2$ and an odd prime $p$. If $B$ is any $p$-block of $\widetilde{\mathfrak{S}}_{n}$, then $B$ contains either no or only spin characters. In the former case, $B$ coincides with a $p$-block of $\mathfrak{S}_{n}$; in the latter, we say that $B$ is a spin block. Similarly, any $p$-block of $\widetilde{\mathcal{A}}_{n}$ contains either no spin character, and coincides with a $p$-block of $\mathcal{A}_{n}$, or only spin characters, and is then called a spin block. The spin blocks of $\widetilde{\mathfrak{S}}_{n}$ and $\widetilde{\mathcal{A}}_{n}$ are described by the following:

Theorem 4.5 (Morris 26, Humphreys [19]). Let $\chi$ and $\psi$ be two spin characters of $\widetilde{\mathfrak{S}}_{n}$, or two spin characters of $\widetilde{\mathcal{A}}_{n}$, labeled by bar partitions $\lambda$ and $\mu$ respectively. Then $\chi$ is of $p$-defect 0 (and thus alone in its $p$-block) if and only if $\lambda$ is a $\bar{p}$-core. If $\lambda$ is not a $\bar{p}$-core, then $\chi$ and $\psi$ belong to the same $p$-block if and only if $\lambda_{(\bar{p})}=\mu_{(\bar{p})}$.

One can therefore define the $\bar{p}$-core of a spin block $B$ and its $\bar{p}$-weight, as well as its sign $\sigma(B)=\sigma\left(\lambda_{(\bar{p})}\right)$ (for any bar partition $\lambda$ labeling some character $\chi \in B$ ).

Theorem 4.6. Let $B$ be any spin p-block of $\widetilde{\mathfrak{S}}_{n}$ of $\bar{p}$-weight $w>0$. Let $\widetilde{G}, \widetilde{H}, \mathcal{C}$ and $\mathcal{D}$ be as in $\$$, and let $\delta_{\bar{p}}(\lambda)$ be the relative sign of a bar partition $\lambda$ introduced by Morris and Olsson in 27.

(i) If $\sigma(B)=1$, then $I: \mathbb{C} B \rightarrow \mathbb{C S I}(\widetilde{G})$ given by

$$
\begin{aligned}
& I\left(\xi_{\lambda}\right)=\delta_{\bar{p}}(\lambda)(-1)^{\left|\lambda^{0}\right|} \psi_{\lambda(\bar{p})} \quad \text { if } \sigma(\lambda)=1, \\
& I\left(\xi_{\lambda}^{ \pm}\right)=\delta_{\bar{p}}(\lambda)(-1)^{\left|\lambda^{0}\right|} \psi_{\left.\lambda^{(} \bar{p}\right)}^{ \pm} \quad \text { if } \sigma(\lambda)=-1 \text {, }
\end{aligned}
$$

is a perfect isometry with respect to p-regular elements of $\widetilde{\mathfrak{S}}_{n}$ and $\mathcal{C}$.

(ii) If $\sigma(B)=-1$, then $I: \mathbb{C} B \rightarrow \mathbb{C S I}(\widetilde{H})$ given by

$$
\begin{array}{ll}
I\left(\xi_{\lambda}\right)=\delta_{\bar{p}}(\lambda)(-1)^{\left|\lambda^{0}\right|} \varphi_{\lambda(\bar{p})} & \text { if } \sigma(\lambda)=1, \\
I\left(\xi_{\lambda}^{ \pm}\right)=\delta_{\bar{p}}(\lambda)(-1)^{\left|\lambda^{0}\right|} \varphi_{\lambda(\bar{p})}^{ \pm} & \text {if } \sigma(\lambda)=-1,
\end{array}
$$

is a perfect isometry with respect to p-regular elements of $\widetilde{\mathfrak{S}}_{n}$ and $\mathcal{D}$.

Proof. (i) By [24, Thm 10.1], the isometry $I_{0}: \mathbb{C} B_{0} \rightarrow \mathbb{C S I}(\widetilde{G})$ given by

$$
\begin{aligned}
& I_{0}\left(\xi_{\mu}\right)=\delta_{\bar{p}}(\mu)(-1)^{w\left(p^{2}-1\right) / 8+w+\left|\mu^{0}\right|} \psi_{\mu^{(\bar{p})}} \quad \text { if } \sigma(\mu)=1, \\
& I_{0}\left(\xi_{\mu}^{ \pm}\right)=\delta_{\bar{p}}(\mu)(-1)^{w\left(p^{2}-1\right) / 8+w+\left|\mu^{0}\right|} \psi_{\mu^{(\bar{p})}}^{ \pm \delta_{\bar{p}}(\mu)(-1)^{w}} \quad \text { if } \sigma(\mu)=-1,
\end{aligned}
$$

is a Broué isometry when $w<p$ and $B_{0}$ is the principal spin $p$-block of $\widetilde{\mathfrak{S}}_{p w}$. Note that, by definition of $\sigma\left(\mu^{0}\right)$, the $\operatorname{sign} \eta_{\bar{p}}(\mu)$ in [24, Thm 10.1] does not in fact depend on $\sigma\left(\mu^{0}\right)$. Now, the proof of [24, Thm 10.1] carries forward to the case where $w \geq p$ to show that $J$ is a perfect isometry with respect to $p$-regular elements of $\widetilde{\mathfrak{S}}_{n}$ and $\mathcal{C}$. Using the fact that, if $J$ is perfect isometry, then so is $-J$, we deduce that $I_{1}=(-1)^{w\left(p^{2}-1\right) / 8+w} I_{0}$ is again a perfect isometry with respect to $p$-regular elements of $\widetilde{\mathfrak{S}}_{n}$ and $\mathcal{C}$. By [7, Thm 4.21], there exists a perfect isometry $I_{2}$ with respect to $p$-regular elements between $B_{0}$ and $B\left(I_{2}\right.$ associates characters of $B_{0}$ and 
$B$ labeled by bar partitions with the same $\bar{p}$-quotient). Furthermore, by composing iteratively perfect isometries of the type described in Theorem 4.4, by Remark 4.1, we construct a perfect isometry $I_{3}: \mathbb{C} B \rightarrow \mathbb{C} B$ with respect to $p$-regular elements and such that $I_{3} \circ I_{2} \circ I_{1}^{-1}: \operatorname{SI}(\widetilde{G}) \rightarrow \mathbb{C} B$ given by $\psi_{\lambda(\bar{p})} \mapsto \xi_{\lambda}$ if $\sigma(\lambda)=1$ and $\psi_{\lambda(\bar{p})}^{ \pm} \mapsto \xi_{\lambda}^{ \pm}$if $\sigma(\lambda)=-1$ is a perfect isometry (by composition and inversion) with respect to $\mathcal{C}$ and $p$-regular elements of $\widetilde{\mathfrak{S}}_{n}$. The result follows from the fact that $I=\left(I_{3} \circ I_{2} \circ I_{1}^{-1}\right)^{-1}$.

(ii) The proof is similar. We start from the Broué isometry between $\operatorname{SI}(\widetilde{H})$ and the principal spin $p$-block $B_{0}^{*}$ of $\widetilde{\mathcal{A}}_{p w}$ given in [24, Thm 10.2], whose proof can be generalized to the case $w \geq p$ to produce a perfect isometry with respect to $\mathcal{D}$ and the set of $p$-regular elements of $\widetilde{\mathcal{A}}_{p w}$. The analogue of the perfect isometry $I_{2}$ of case (i) is the perfect isometry $B_{0}^{*} \rightarrow B$ given in [7, Thm 4.21].

Remark 4.7. If $w<p$, then the same proof, but using Lemma4.2, Remark 4.3 and Theorem 4.4, shows that the isometries given in Theorem 4.6 are Broué isometries.

Corollary 4.8. If $B$ is any spin $p$-block of $\widetilde{\mathfrak{S}}_{n}$ or of $\widetilde{\mathcal{A}}_{n}$ of $\bar{p}$-weight $w$, then the set of characters of $B$ labeled by bar partitions $\lambda$ such that $\lambda^{0}=\emptyset$ is a p-basic set of $B$.

Proof. In the case of $\widetilde{\mathfrak{S}}_{n}$, this follows from Theorem 4.6, Theorems 3.3 and 3.4, and [9. Prop 2.2] when $w>0$. If $w=0$, then $B$ contains a single character and the result is obvious. If $B$ is spin $p$-block of $\widetilde{\mathcal{A}}_{n}$, then [7, Thm 4.21] provides a perfect isometry from $B$ to a spin $p$-block of same $\bar{p}$-weight $w$ of some $\widetilde{\mathfrak{S}}_{m}$, and which preserves the labeling of characters. The result then follows from the first part of the proof and [9, Prop 2.2].

Any spin $p$-block of $\widetilde{\mathfrak{S}}_{n}$ or of $\widetilde{\mathcal{A}}_{n}$ has a $p$-basic set by Corollary 4.8, and any other block has a $p$-basic set by [9]. Hence, $\widetilde{\mathfrak{S}}_{n}$ and $\widetilde{\mathcal{A}}_{n}$ have $p$-basic sets, which proves Theorem 1.1 .

\section{Some FURTHER REMARKS}

First notice that, by construction, the $p$-basic set $b_{S}$ of $\widetilde{\mathfrak{S}}_{n}$ we produce is stable under tensor product by $\varepsilon$. Furthermore, when restricted to $\widetilde{\mathcal{A}}_{n}$, it gives our $p$-basic set $b_{A}$ of $\widetilde{\mathcal{A}}_{n}$, that is, the elements of $b_{A}$ are exactly the irreducible constituents of the restrictions to $\widetilde{\mathcal{A}}_{n}$ of the elements of $b_{S}$.

By Remark 3.5, and using the perfect isometry (constructed in [7, Thm 5.12]) between a $p$-block of $\mathcal{A}_{n}$ and $\operatorname{Irr}(H)$ with respect to $p$-regular elements of $\mathcal{A}_{n}$ and $\theta(\mathcal{D})$, one can directly obtain the $p$-basic set of $\mathcal{A}_{n}$ constructed in [9, Thm 5.2]. The advantage of this new method is that we need not worry about Property (2) in [9, Thm 1.1] which is automatically satisfied.

If $B$ is any spin $p$-block of $\widetilde{\mathfrak{S}}_{n}$ or $\widetilde{\mathcal{A}}_{n}$, then the characters in the $p$-basic set $b$ of $B$ obtained in Corollary 4.8 are labeled by bar partitions $\lambda$ such that $\lambda^{(\bar{p})}=\left(\lambda^{0}=\right.$ $\left.\emptyset, \lambda^{1}, \ldots, \lambda^{(p-1) / 2}\right)$, or, equivalently, by the $(p-1) / 2$-quotients of $w$. Furthermore, for each such $\lambda$, one has

$$
\sigma\left(\lambda^{(\bar{p})}\right)=(-1)^{w-\ell\left(\lambda^{0}\right)}=(-1)^{w},
$$

so that all quotients label two spin characters of $\widetilde{\mathfrak{S}}_{n}$ and one of $\widetilde{\mathcal{A}}_{n}$ is $w$ is odd and $\sigma(B)=1$, or if $w$ is even and $\sigma(B)=-1$, and one spin character of $\widetilde{\mathfrak{S}}_{n}$ and two of 
$\widetilde{\mathcal{A}}_{n}$ is $w$ is odd and $\sigma(B)=-1$, or if $w$ is even and $\sigma(B)=1$. In particular, all the characters in $b$ are self-associate, or none is. Since $b$ is $\varepsilon$-stable, the proof of [9, Prop 6.1] implies that the same is true for the set of irreducible Brauer characters in $B$, that is, all are self-associate or none is. As a consequence, we also obtain a formula for the number of irreducible Brauer characters in $B$. We therefore recover results of Olsson (see [29, Prop (3.21) and (3.22)]) obtained by sophisticated methods.

We end this paper with some considerations about what we like to call Olsson's Principle. Roughly speaking, Olsson's Principle predicts that, for any statement which holds for $\mathfrak{S}_{n}$, an analogous spin statement must hold for $\widetilde{\mathfrak{S}}_{n}$, even though one might have to be careful with signs along the proof.

We now present a few illustrations of this principle which appear in our work. Recall that the irreducible characters of $\mathfrak{S}_{n}$ are parametrized by the set of partitions of $n$. For any prime $p$, a partition $\lambda$ of $n$ is uniquely determined by its $p$-core $\lambda_{(p)}$ and its $p$-quotient $\lambda^{(p)}=\left(\lambda^{1}, \ldots, \lambda^{p}\right)$ which is a $p$-tuple of partitions whose sizes add up to $w$, the $p$-weight of $\lambda$. The celebrated Nakayama Conjecture states that two irreducible characters $\chi_{\lambda}$ and $\chi_{\mu}$ of $\mathfrak{S}_{n}$ belong to the same $p$-block if and only if $\lambda_{(p)}=\mu_{(p)}$. The spin analogue is given by the Morris Conjecture (Theorem 4.5). Now, the $p$-basic set of $\mathfrak{S}_{n}$ constructed in [9] consists of those characters which are labeled by partitions $\lambda$ with $\lambda^{(p+1) / 2}=\emptyset$, i.e. without symmetric diagonal $(p)$-hooks. The spin analogue of this is the set of bar partitions $\lambda$ of $n$ such that $\lambda^{0}=\emptyset$, i.e. without parts divisible by $p$. The link between these two properties is enlightened by the doubling construction presented in 28. Via this process, a bar partition $\lambda$ of $n$ is associated to a partition $D(\lambda)$ of $2 n$ such that $\lambda^{0}=\emptyset$ if and only if $D(\lambda)^{(p+1) / 2}=\emptyset$.

The way the irreducible spin characters of $\widetilde{G}$ can be labeled by $\bar{p}$-quotients of $w$, just like the irreducible characters of $G$ are labeled by $p$-quotients of $w$ is another example of Olsson's Principle. It should therefore not come as a surprise that the perfect isometry of Theorem 4.6 (which associates spin characters of $\widetilde{\mathfrak{S}}_{n}$ and $\widetilde{G}$ corresponding to the same $\bar{p}$-quotient of $w$ ), is precisely the spin analogue of the perfect isometry of [9, Thm 3.6] (which associates characters of $\mathfrak{S}_{n}$ and $G$ corresponding to the same $p$-quotient of $w$ ), including the sign $\delta_{\bar{p}}(\lambda)(-1)^{\left|\lambda^{0}\right|}$ which is the spin analogue of $\delta_{p}(\lambda)(-1)^{\left|\lambda^{(p+1) / 2}\right|}$.

By the same token, the basic set of $\operatorname{SI}(\widetilde{G})$ we construct in Theorem 3.3 is the spin analogue of that of $G$ given in [9, Thm 4.3].

It should be noted that, while Olsson's Principle can be an inspiration for results about $\widetilde{\mathfrak{S}}_{n}$, it is by no means a proof. However, it can be a useful guide to indicate what the proof should be. This is indeed the narrative behind the sequence given by [7], [24] and the present paper to generalize to the spin case the proof of [9], whose result's spin analogue was inspired to the authors by Olsson's Principle several years ago.

Acknowledgements. Part of this work was done at the CIRM in Luminy during a research in pairs stay. The authors wish to thank the CIRM gratefully for their financial and logistical support. The first author is supported by Agence Nationale de la Recherche Projet ACORT ANR-12-JS01-0003. The second author also acknowledges financial support from the Engineering and Physical Sciences Research Council grant Combinatorial Representation Theory EP/M019292/1. 


\section{REFERENCES}

[1] G. E. Andrews, C. Bessenrodt, and J. B. Olsson. Partition identities and labels for some modular characters. Trans. Amer. Math. Soc., 344(2):597-615, 1994.

[2] C. Bessenrodt. A 2-block splitting in alternating groups. Algebra Number Theory, 3(7):835846, 2009.

[3] C. Bessenrodt, A. Morris, and J. B. Olsson. Decomposition matrices for spin characters of symmetric groups at characteristic 3. J. Algebra, 164(1):146-172, 1994.

[4] C. Bessenrodt and J. B. Olsson. The 2-blocks of the covering groups of the symmetric groups. Adv. Math., 129(2):261-300, 1997.

[5] M. Broué. Isométries parfaites, types de blocs, catégories dérivées. Astérisque, (181-182):6192,1990

[6] O. Brunat. Basic sets in defining characteristic for general linear groups of small rank. J. Pure Appl. Algebra, doi:10.1016/j.jpaa.2008.09.006, 2008.

[7] O. Brunat and J. Gramain. Perfect isometries and murnaghan-nakayama rules. To appear in Transaction of the AMS.

[8] O. Brunat and J. Gramain. A 2-basic set of the alternating group. Arch. Math. (Basel), 94(4):301-309, 2010.

[9] O. Brunat and J. Gramain. A basic set for the alternating group. J. Reine Angew. Math., 641:177-202, 2010.

[10] D. Denoncin. Stable basic set for finite special linear and unitary groups. preprint.

[11] W. Feit. The representation theory of finite groups, volume 25 of North-Holland Mathematical Library. North-Holland Publishing Co., Amsterdam, 1982.

[12] M. Geck. Irreducible Brauer characters of the 3-dimensional special unitary groups in nondefining characteristic. Comm. Algebra, 18(2):563-584, 1990.

[13] M. Geck. Basic sets of Brauer characters of finite groups of Lie type. II. J. London Math. Soc. (2), 47(2):255-268, 1993.

[14] M. Geck. Basic sets of Brauer characters of finite groups of Lie type. III. Manuscripta Math., 85(2):195-216, 1994.

[15] M. Geck and G. Hiss. Basic sets of Brauer characters of finite groups of Lie type. J. Reine Angew. Math., 418:173-188, 1991.

[16] J.-B. Gramain. The Isaacs-Navarro conjecture for covering groups of the symmetric and alternating groups in odd characteristic. J. Algebraic Combin., 34(3):401-426, 2011.

[17] G. Hiss. On the decomposition numbers of $G_{2}(q)$. J. Algebra, 120(2):339-360, 1989.

[18] J. F. Humphreys. Blocks of projective representations of the symmetric groups. J. London Math. Soc. (2), 33(3):441-452, 1986.

[19] J. F. Humphreys. Blocks of projective representations of the symmetric groups. J. London Math. Soc. (2), 32:441-452, 1986.

[20] I. Isaacs. Character theory of finite groups. Academic Press [Harcourt Brace Jovanovich Publishers], New York, 1976. Pure and Applied Mathematics, No. 69.

[21] G. James and A. Kerber. The representation theory of the symmetric group, volume 16 of Encyclopedia of Mathematics and its Applications. Addison-Wesley Publishing Co., Reading, Mass., 1981.

[22] A. S. Kleshchev and P. H. Tiep. Representations of finite special linear groups in non-defining characteristic. Adv. Math., 220(2):478-504, 2009.

[23] B. Külshammer, J. B. Olsson, and G. R. Robinson. Generalized blocks for symmetric groups. Invent. Math., 151(3):513-552, 2003.

[24] M. Livesey. Broué's Perfect Isometry Conjecture Holds for the Double Covers of the Symmetric and Alternating Groups. Algebr. Represent. Theory, DOI 10.1007/s10468-016-9599-2, 2016.

[25] G. O. Michler and J. B. Olsson. The Alperin-McKay conjecture holds in the covering groups of symmetric and alternating groups, $p \neq 2$. J. Reine Angew. Math., 405:78-111, 1990.

[26] A. O. Morris. The spin representation of the symmetric group. Canad. J. Math., 17:543-549, 1965.

[27] A. O. Morris and J. B. Olsson. On p-quotients for spin characters. J. Algebra, 119(1):51-82, 1988.

[28] A. O. Morris and A. K. Yaseen. Some combinatorial results involving shifted Young diagrams. Math. Proc. Camb. Phil. Soc., 99:23-31, 1986. 
[29] J. B. Olsson. The number of modular characters in certain p-blocks. Proc. London Math. Soc. (3), 65(2):245-265, 1992.

[30] J. B. Olsson. Combinatorics and Representations of Finite Groups. Vorlesungen aus dem Fachbereich Mathematik der Universität GH Essen, Helf 20, 1993.

[31] I. Schur. Über die Darstellung der symmetrischen und der alternierenden Gruppe durch gebrochene lineare Substitutionen. J. Reine Angew. Math., 139:155-250, 1911.

Université Paris-Diderot Paris 7, Institut de mathématiques de Jussieu - Paris Rive Gauche, UFR de mathématiques, Case 7012, 75205 Paris Cedex 13, France.

E-mail address: brunat@math.univ-paris-diderot.fr

Institute of Mathematics, University of Aberdeen, King's College, Fraser Noble Building, Aberdeen AB24 3UE, UK

E-mail address: jbgramain@abdn.ac.uk 